\title{
A Bio-inspired Framework for Highly Efficient Structural Health Monitoring and Vibration Analysis
}

\author{
Maria-Giovanna Masciotta ${ }^{1}$, Alberto Barontini' ${ }^{2}$, Luís F. Ramos ${ }^{3}$, Paulo Amado-Mendes ${ }^{4}$, Paulo B. Lourenço ${ }^{5}$ \\ ${ }^{1}$ Postdoc Researcher, ISISE, Department of Civil Engineering, University of Minho - Guimarães, Portugal. \\ ${ }^{2}$ Ph.D. student, ISISE, Department of Civil Engineering, University of Minho - Guimarães, Portugal. \\ ${ }^{3}$ Assistant Professor, ISISE, Department of Civil Engineering, University of Minho - Guimarães, Portugal. \\ ${ }^{4}$ Assistant Professor, ISISE, Department of Civil Engineering, University of Coimbra - Coimbra, Portugal. \\ ${ }^{5}$ Full Profess or, ISISE, Department of Civil Engineering, Univers ity of Minho - Guimarães, Portugal.
}

\begin{abstract}
Civil engineering structures are continuously exposed to the risk of damage whether due to ageing effects, excessive live loa ds or extreme events, such as earthquakes, blasts and cyclones. If not readily identified, damage will inevitably compromise the structural integrity, leading the systemto stop operating and undergo in-depth interventions. The economic and social impacts associated with such an adverse condition can be significant, therefore effective methods able to early identify structural vulnerabilities are needed for these systems to keep meeting the required life-safety standards and avoid the impairment of their normal function. In this context, vibration-based analys is approaches play a leading role as they allow to detect structuralfaults which lie beneath the surface of the structure by identifying and quantifying anomalous changes in the system's inherent vibration characteristics. However, although the considerable degree of maturity attained within the fields of experimental vibration analysis (EVA) and structural health monitoring (SHM), several technical issues still need to be addressed in order to ensure the successfulimplementation of these powerful tools for damage identification purposes.
\end{abstract}

The scope of this paper is to present a bio-inspired framework for optimal structural health monitoring and vibration analysis. After a critical overview on current methods and tools, three main sources of bio-inspiration are described along with the relative algorithms derived for SHM applications. It is shown how uncovering the general principles behind the functioning of selected biological systems can foster the development of efficient solutions to the technical conflicts of actual SHM architectures and lead to new sensing paradigms for optimal network to pology and sensors location. A compatibility-matrix is proposed to help compare biological and SHM systems and discriminate desired from unwanted features. Such a framework will ultimately assist in seeking for the most suitable nature-ins pired solutions for more accurate condition screening and robust vibration analysis.

Keywords: Structural health monitoring, vibration analysis, bio-inspired framework, compatibility-matrix, optimal network topology.

\section{INTRODUCTION}

The potentialfor significant life-safety and economic benefits as sociated with structuralhealth monitoring (SHM) methods has considerably boosted their implementation into the engineering practice [1]. Unlike traditional visual inspection methods, which can be subjective in nature and result insufficient when structural defects are not visible to human eyes, SHM systems may allow to catch even subtle structural changes, thereby allowing for more accurate condition screenings and continuous check-ups of the systemhealth. Notwithstanding that, infrastructure managers often prefer experience-based solutions to SHMweighed approaches, as the misuse and abuse of the latter can lead to inappropriate corrective actions and irremediably jeopardize the structural safety, resulting in considerable loss of economical and human resources. Hence the necessity to define new high-performance SHM strategies and to adopt a methodical framework for the successful implementation of such strategies in the engineering practice. Simultaneously, specific efforts have to be made to educate owners and raise their awareness about the consequences of poor structural health and the benefits that can be gained over time from the adoption of SHM-based preventive actions with respect to more invasive belated repair measures. In order to move forward in this direction, several problems as sociated with current SHM technologies must be overcome. To this end, in the last years, engineers have turned to biology for sources of inspiration, giving rise to new sensing paradigms for highly efficient SHM systems . Although the youth of this field, the amount of research related to bio-inspired systems is rapidly increasing both at academic and industrial levels. By reviewing what has been done so far in terms of bio-inspired SHM and by outlining a methodical 
framework for the development of new bio-ins pired sensing patterns, the present paper wants to provide a useful contribution to the research community working on this topic.

\section{CRITICAL APPRAISAL OF CURRENT SHM METHODS AND TOOLS}

\section{General overview of traditional and modern SHM systems}

The field of SHM is very wide and encompasses severaltechniques classified according to various criteria. In what concems vibration-based SHM techniques, two categories can be distinguished: traditional and modern. Traditional techniques rely on tethered sensing systems which exploit a certain number of sensors (e.g. accelerometers, displacement transducers, strain gauges, and the like) distributed over the structure and wired back through coaxial cables to a centralized DAQ systemfor data storing and processing (Figure 1a). Although reliable in communication, the use of cables drags behind various problems, in particular the high cost per sensing node channel and the considerable amount of time and manual work necessary to install and maintain dense sets of sensors. The large number of wires, fibre optic cables, or other physical transmission media may be prohibitive, especially in case of large-scale structures such as long-span bridges or tall buildings [2]. Moreover, wires need to be isolated from the harsh environment to avoid measurement corruption, tearing, rodent nibbling, thus causing a further increase in the cost and time of installation. These reasons explain why tethered systems usually lack a high spatial density of sensors, which turns out to be very important for damage detection purposes as it allows to achieve greater monitoring fidelity.

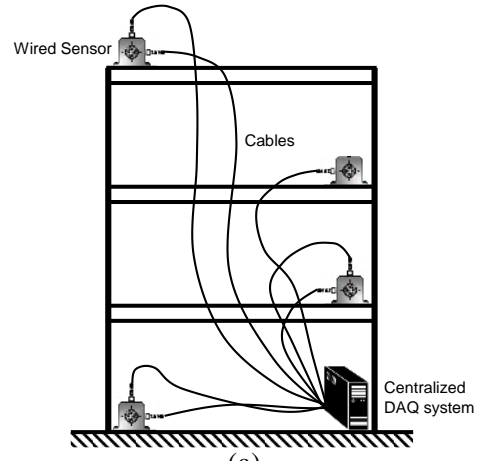

(a)

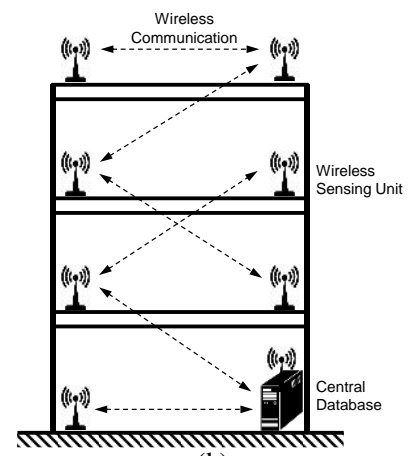

(b)

Figure 1. Configuration of SHM systems: (a) tethered versus (b) wireless (adapted from [26]).

The limitations of traditional monitoring techniques have led structural engineering researchers to seek for alternative and more modern approaches in order to perform many of the tasks associated with the health monitoring of civil structures. Such approaches are based on the use of sensing units that embody wireles s communications and mobile computing elements so as to deliver a relatively inexpensive sensor platform [3] with distributed and no physically linked data acquisition nodes (Figure 1b). Each wireless sensor is provided with an on-board computational core responsible for data collection, self-interrogation of measurement data and wireless communication within the sensor network. Thanks to the rapid advances in sensors technology and the decreasing cost of computing and communication systems, diverse wireless monitoring platforms have been designed and tested on different applications [3]-[4]. Such systems feature a cheaper and quicker installation combined with a superior performance, thereby resulting valuable low-cost substitutes for tethered monitoring systems. In spite of that, several challenges and bottlenecks must still be overcome to fully realize the potential of wireless SHM architecture [3]. Hence, further research is required in this field.

\section{Key problems and challenges}

Among the wide number of issues to address are the over instrumentation and data inundation in SHM systems due to inappropriate sensors selection and location. In this regard, reliable algorithms for optimal sensors placement should be developed and lossless data compression techniques must be applied to reduce the flow of sensorinformation as well as face the limited capacity of wireless sensing units. Another is sue to deal with is the data transmission within the wireless monitoring system which often results inaccurate and incomplete because of limited communication bandwidth, radio interference and path loss. The energy consumption and the operational life of the monitoring system are also key aspects that need to be tackled. The power supplies of wireless sensors are indeed finite and the replacement of batteries in locations that are not easily accessible is often difficult. To trade off functionality and power consumption, the design of a flexible network management software combining sleep/wake cycles with threshold detection may be of great help. Although this would imply an $a$ priori hierarchical planning of the wireless network topology, improving the entire Wireless Sensor Network (WSN) architecture before its implementation will ultimately upgrade the SHM system's performance and lead to a clever multi-level screening reducing both operation and maintenance costs. In addition, some extra efforts should be made to enhance the time synchronization of sensors in order to align data streams at different locations [5]. Last but not least issue is the influence of 
ambient factors and background noise on the vibration response of structures in operation conditions and the consequent necessity to provide wireless sensing units with computing procedures capable to autonomously spot and filter out these effects, which may otherwise risk to mask changes due to damage occurrence.

In light of the above considerations, it is clear that architectural and networking is sues are not independent and cannot be addressed in isolation. A holistic approach, distinct from previous SHM strategies, is therefore needed.

\section{BIOMIMICRY AND STRUCTURAL HEALTH MONITORING}

\section{Background on biomimetic concepts}

To master imposing engineering challenges such as the ones mentioned previously, engineers have recently turned to biology for sources of inspiration, giving rise to a new field called biomimetics, biomimicry or bio-inspired engineering. From an etymological viewpoint, the term biomimetics comes from the Greek words bios, meaning life, and mimesis, meaning imitation. The name was coined in the 1950s by the polymath Otto Schmitt [6], whose doctoral research focused on the development of a physical device that explicitly mimicked the electrical action of a nerve. The chief idea of biomimetics is that Nature has already engineered superior, ingenious and sustainable systems which have benefited from millions of years of evolution and optimization and whose capabilities far surpass many of currently available technologies. Thus, the main scope of this new born discipline is to identify potential processes and mechanisms in biological systems and emulate them in order to develop innovative and efficient nature-inspired solutions in different fields and applications.

It is worth noting that mankind has been taking hints from biology for centuries. One of the earliest although unsuccessful attempt of biomimicry dates back to the $15^{\text {th }}$ century, when Leonardo Da Vinci tried to build a "flying machine" based on the study of both anatomy and flying mechanisms of winged animals. Lately, with the technological progress and the integration of life and natural sciences with engineering and physical sciences, biomimetics has aroused interest across numerous disciplines and bio-inspired concepts have been assimilated into several areas, including material science, robotics and computer science. The most famous example of biomimicry is the hook-and-loop fastener, also known as Velcro [7], which was conceived in the late 1940s by the Swis s engineer George de Mestral after examining under a microscope the burs stuck to his clothes and his dog's fur after returning from a hunting trip. Evidence of the benefits yielded by bio-inspired systems has been shown in various practical applications, including structures, aerodynamics of vehicles, highly efficient devices and apparatus [8]-[10]. Today, the word biomimetics is used in all contexts that involve the transfer of skills or information from biology to applied science.

\section{Sources of bio-inspiration in SHM and relevant algorithms}

Given its success in many different areas of engineering, biomimetics is recently being explored for SHM purposes, guiding in the definition of nature-inspired sensing patterns for the development of highly efficient wireless sensor networks. So far, scientists seem to be particularly prone to investigate the processing mechanisms featured by biological sensory systems, due to the simplistic individual functional unit, the neuron, which forms sophisticated architectures with other units to convey information to the brain in real time [11]. This section gives a brief review of three of the main sources of bio-inspiration that have already found actuation in SHM (i.e. central nervous system, auditory system, and immune system) along with the most relevant bio-inspired algorithms derived for SHM applications. The goal is to identify the aspects upon which further research must be addressed for the advancement of the subject and to lead the way for the definition of a systematic framework for new bio-ins pired sensing paradigms in the context of SHM.

\section{Central nervous system}

The central nervous system(CNS) is one of the first sources of bio-inspiration that has been drawing the attention of engineers and mathematicians, especially in what concerns its adaptive architecture and parallel processing capabilities. The centre of the nervous system is the brain, which operates in combination with the spinal cord through a network of electrically excitable cells called neurons. Neurons can be of different types, but they all look alike in morphology and architecture. A typical neuron consists of three regions, namely the cell body, the axon and the dendrites. The cell body (or soma) analyses the incoming signals; the axon carries the signals to other neurons; and the dendrites receive the electrochemical signals from other cells' axons (Figure 2). Note that neurons are not continuous, yet they communicate with one another through specific structures called synapses (from the Greek synapsis, meaning "conjunction"), each consisting of a presynaptic part located at the end of the axon and a postsynaptic part usually located upon another neuron's dendrite. Throughout life synapses can be subject to alterations, e.g. short-term physiologicalchanges can modify the effectiveness of the synapses whereas long-termphysiological changes can lead to modifications of the anatomy of the connection itself.

Each neuron is capable of encoding the information received from external input signals into all-or-none discrete electrochemical pulses called 'action potentials' or spike train, whose firing rate is linked to the amplitude and frequency of the input [12]. If the action potentials surpass a certain threshold, an impulse is sent down the axon's terminal cell, where 
neurotransmitters are released to transmit the impulse to the receptors located on the postsynaptic neurons. This yields a complete signal processing and information integration through complex networks of neurons which are able to adapt over time depending on the input stimuli and to autonomously make sophisticated decisions [13]. It is remarked that the CNS enables high-speed simultaneous processing of analogous information either in different groups of neurons or in differe nt neurons of the same pathway, being endowed with parallel processing capabilities. Furthermore, to minimize energy expenses while maintaining fast conduction, many neurons have insulating myelin sheaths around their axons. Not to mention the importance of the glial cells in providing support and nutrition to neurons, insulating themelectrically, maintaining homeostasis, destroying pathogens and participating in the signal transmission within the nervous system. An extensive description of the physiology of the CNS can be found in [13].

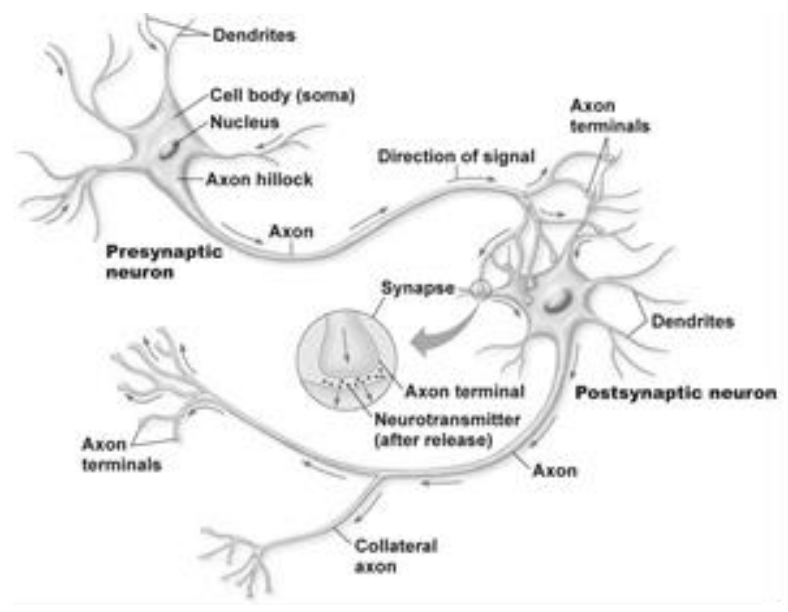

Figure 2. Schematic architecture of two neurons (droualb.faculty.mjc.edu).

Taking cue from nature, Artificial Neural Networks (ANNs) were developed in the early 1940's as an attempt to mathematically represent the basic physiology of a network of neurons for computer software purposes. The first mathematical model was developed by McCulloch and Pitts [14] who conceived the neuron as an extremely simplified processing unit firing only if the weighted sum of the inputs exceeded a certain threshold value $\alpha$. Following this first work, several novel learning algorithms were conceived, leading to the development of numerous ANN-based methods [15]-[16]. In what concerns SHM, the most used neural network architectures included the multilayer perceptron (MLP) with sigmoidal activation function [17], the counter propagation neural network [18], the probabilistic neural network [19], and the recurrent neural network [20]. Real applications of ANN for SHM purposes have been reviewed in literature [21], pointing out the efficiency of neural networks as non-model-based methods for damage identification in the presence of training sets (supervised learning). Nevertheless, neural networks have also shown good results in identifying damage patterns not belonging to training sets (unsupervised learning), which is essentialfor monitoring issues [22].

Beyond the achievements, it should be stressed that the original intent of ANNs was to mimic the processing mechanisms employed by the nervous system, but their evolution finally diverged from the primary source of inspiration. For instance, the architectural design of ANNs has no connection with the original human neural circuit and often ANNs are used in combination with other methods, further deviating from the initial biological principles.

\section{$\underline{\text { Auditory system }}$}

Recently, a new source of inspiration for robust SHM has been chosen with the purpose of returning to the original principles of biological sensory systems. This is the mammalian auditory system, a subsystemof the sensory system, which boasts realtime spectral decomposition and high compression capabilities that render it an ideal source of inspiration for improving the performance of structural monitoring systems [23]. The mammalian auditory systemrelies on a few operational principles. The sound wave is received at the ear canal, or outer ear, is passed to the middle ear through the ear drum, and is transmitted to the inner ear by means of induced vibrations at the oval window, which is directly connected to the base of the cochlea, the main signal processing unit of the auditory system [24]. The cochlea is a snail-shaped tube partitioned in three fluid-filled cavities by two membranes, i.e. the Reissner's membrane and the basilar membrane (Figure 3a-b). The basilar membrane features a geometric configuration and a mechanical stiffness that change across its length, therefore the natural frequency of this membrane also varies across its development. The deflections and resonant frequencies of the basilar membrane are correlated and tuned to the external sound wave [23]. Based on the vibrating subsections of this membrane (Figure 3c), the cochlea instantaneously maps out the frequency content of the signal and conveys the perceived stimulus to higher levels of the auditory cortex via neural signals [25]. In detail, the stereocilia and as sociated inner hair cells (IHCs) of the organ of Corti, which runs 
on top of the basilar membrane, are responsible for encoding the membrane's motion into electrical spikes ('action potentials') which are transmitted to the CNS [24]. Being each electrical spike an all-or-none event, the spike will only fire if a threshold stimulus is reached and it will be part of a series of pulses that jointly encode information about the perceived stimulus. By using this unique method of spectral decomposition, the auditory system is able to attain an impressive auditory range while maintaining near real-time processing capabilities.

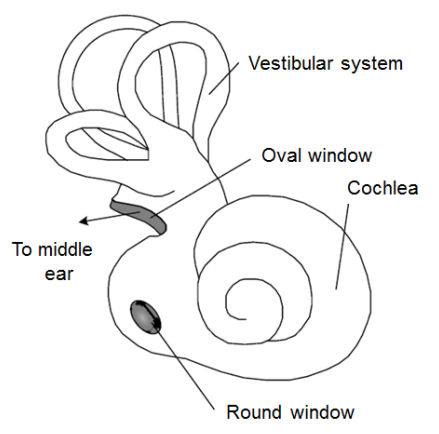

(a)

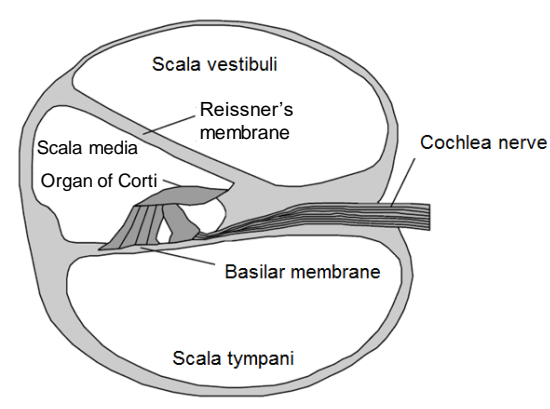

(b)

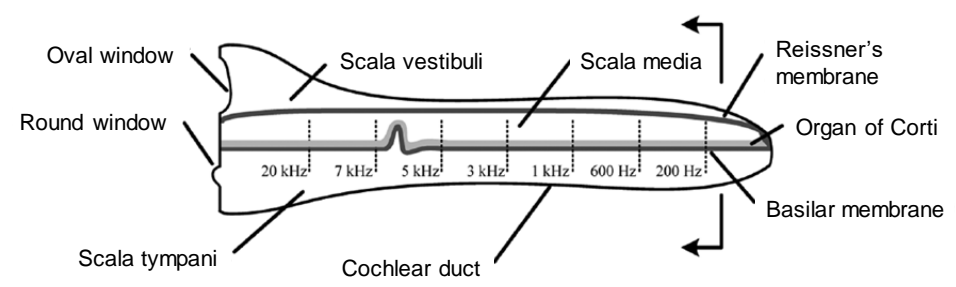

(c)

Figure 3. Schematic representation of the mammalian auditory system: (a) inner ear; (b) cross-section of the cochlea; and (c) uncoiled cochlea indicating the vibrating subsections of the basilarmembrane [25].

Based on the operational principles of the auditory system, Peckens and Lynch proposed a bio-inspired sensor [23] for structural monitoring applications. This sensor was conceived by mimicking the main processing mechanis ms of the mammalian cochlea: 1) spectral decomposition by the basilar membrane; and 2) linear encoding of peak values and data compression by the inner hair cells. These mechanisms were translated into an engineered cochlea-inspired sensor system provided with a band-pass filter bank for frequency decomposition of the input signal and an ultralow power microcontroller for nearly real-time peak picking and linear encoding of the filtered signals [23]. The properties of the CNS, combined with the signal processing capabilities of the auditory system, were interpreted from an engineering viewpoint and their functionalities were ultimately applied for the enhancement of structural monitoring systems through the development of an efficient WSN architecture based on the biomimicry of the CNS. By emulating the interactions between neurons and muscles, a bio-inspired control algorithm was formulated and validated on a four-story shear mock-up [26].

The cochlea-inspired sensor did succeed in overcoming many of the technical limitations of traditional wireless sensornodes, marking the beginning of a new era. However, there is still room for improvement, especially in terms of reduction of overhead information as well as learning and adaptability capabilities, characteristics seen within biological systems but not yet implemented. Additionally, a real application on a full scale structure is necessary to demonstrate the real-time monitoring and control capabilities of this bio-inspired technology in environments harsher than those afforded by controlled laboratory experiments.

\section{$\underline{\text { Immune system }}$}

The human immune systemis another source from which scientists are taking inspiration for building up optimal bio-inspired solutions in several fields. This systemis formed by different biological structures and processes that aimat protecting the body against harmful microorganisms and infections. The first objective of the immune system is to differentiate between self and non-self entities. The former belong to the same organism of the immune system, whereas the latter are potential diseasecausing agents, such as viruses, bacteria, parasites, etc., scientifically referred to as pathogens. The immune system operates by unfolding a multilevel defence of increasing specificity [27]: Level 1 - external and internal epithelial surfaces of the body; Level 2 - innate immune system; and Level 3 - adaptive immune system. The first two levels of defence are able to prevent most of infections, but when pathogens evade the innate immune response, the last level comes into play (Figure 4). Vital for the adaptive response of the immune system are the lymphocytes, i.e. cells produced in the bone marrow that mature either within the thymus ( $\mathrm{T}$ lymphocytes) or within the bone marrow itself (B lymphocytes). Each lymphocyte exhibits receptors able 
to recognize and bind with specific molecular patterns called antigens [27]. The strength of this bond is named 'affinity'. Antigen specificity permits the generation of responses that are tailored to certain pathogens. In detail, to combat infections, B lymphocytes differentiate into effector B-cells producing antibodies, proteins able to identify and neutralize pathogens after binding to a specific antigen; whereas T lymphocytes differentiate into effector T-cells that kill infected cells or activate other cells. After defeating the disease, most of effector cells die, but the few cells that survive ('memory cells') memorize the tailored response of the immune system ('immunological memory') so as to unfold a rapid and more effective response in case of a new contact with the same pathogen [27].

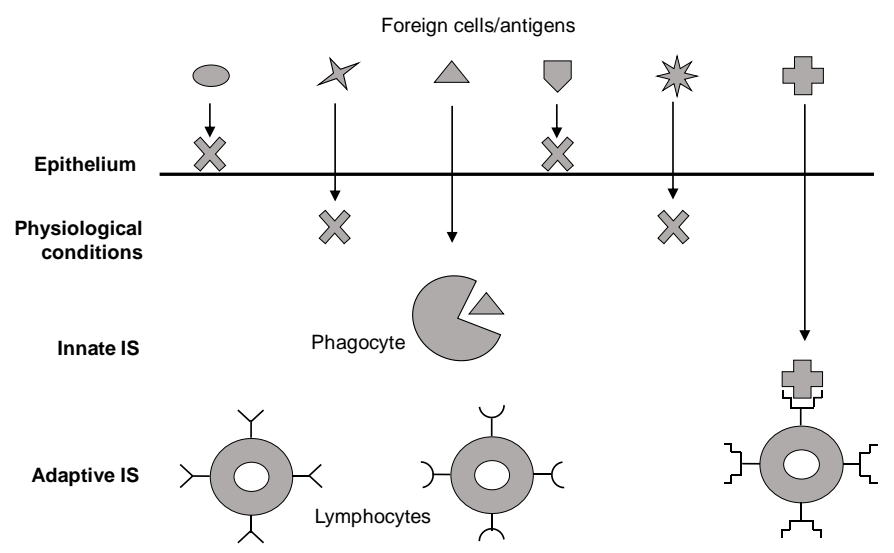

Figure 4. Schematic representation of the multilevel defence structure of the immune system (adapted from [40]).

Inspired by immunology, several adaptive problem-solving methods, and consequently algorithms, have been developed and collected under the name of Artificial Immune Systems (AISs) [28]. Among the main algorithms are negative and positive selection algorithms, resulting from the evolution of the Self/Non-self discrimination theory formulated by Joshua Lederberg [29]. The main goal of this class of algorithms is to achieve a first identification of an emerging damage scenario, just by recognizing it as the cause of an abnormal pattern of features. Based on the clonal selection theory developed in the late $1970 \mathrm{~s}$ by Burnet [30], de Castro and Von Zuben [31] proposed the first clonal selection algorithm, named CLONALG. Different approaches have been suggested over time to improve the reliability of the initial clonal selection algorithm. Detailed reviews of both classes of algorithms can be found in literature [32]. A considerable step forward in the development of a bio-inspired theory of the immune systemwas made by Jerne and Hoffmann [33], who tried to formalize the interacting network of immune system cells by leveraging their ability to mutually recognize each other and to create a dynamic system of self-stimulated elements, even in the absence of external aggressions. The numerical formulation for activation/suppres sion of cells within the network was formalized into a model only some years later by Farmer et al. [34]. This model inspired the first Artificial Immune Network algorithm proposed by Ishida [35] in the 1990s.

AIS-based methods present several strengths, particularly in terms of flexibility, adaptability and learning capabilities. Indeed, the advantages of immune-inspired methods have fostered their use in different fields, such as Computer and Network Security, Fault Detection, Pattern Recognition, Data Mining Clustering and Filtering, Machine Learning, Function Optimization, and many others [36]. AIS architectures have also been developed and successfully applied in the field of mechanical engineering for condition monitoring purposes [37], but just a few works have extended the results to SHM applications in civil engineering. Some examples in the field of immune-inspired SHM are the Artificial Immune Pattern Recognition (AIPR) method formulated by Chen and Zang [38]; the damage detection model-based AIS algorithm with weighted attributes developed by Abbasidoust et al. [39]; and the very recent work of Anaya et al. [40], who presented an active systemfor structuraldamage detection based on the use of piezoelectric transducers acting either as actuators or sensors. Being strongly reliable in tackling optimization problems, clonal selection and immune network theories have also inspired algorithms for improving the sensor deployment in the monitoring of civil engineering systems [41]. However, when dealing with damage identification problems, most of these algorithms allow to identify damage location and extent only if they are correctly trained to recognize the features of a give $n$ damage scenario or when, in case of supervised process, a label to the emerged damage is as signed for future recognition. This is one of the main is sues to address to truly benefit from immune-inspired techniques for both supervised and unsupervised SHM.

Aiming at enhancing setting and effectiveness of the method, the evolution of AISs did ultimately deviate from the original source of inspiration, as seen for ANNs. For instance, in several cases, AIS-based algorithms are combined with non-bioinspired models [42], preventing completely from the actuation of the initial bio-inspired paradigm. 


\section{A FRAMEW ORK FOR NEW BIO-INSPIRED PATTERNS IN SHM}

As shown in the previous section, a few significant and successfulcontributions to the development of bio-inspired solutions have been produced in the last decade, aimed at overcoming most of the problems of actual SHM architectures. Still, several technical aspects remain not completely addressed. It is clear that by uncovering the general principles behind the functioning of selected biological systems, new sensing and control methods can be formulated from which current engineered monitoring systems may yet largely benefit. But, to pursue that, it is necessary to unfold a different strategy. Most bio-inspired paradigms have been conceived for reasons other than health monitoring and structural con trol. Hitherto, the main idea was to browse through biological literature and try to adjust some potential prototype for emulation into some artificial system which could likely turn out to be useful for SHM applications. On the contrary, a good strategy must first focus on the identification of all desirable properties and functions a robust SHM systemmust be provided with. Only after having a clear definition of the desired SHM features, one should look for functional analogies in biology in order to understand how nature has addressed similar instances, and eventually how to model an artificial systemon a very complex natural prototype to imitate its functional principles as closely as possible.

Table 1. Compatibility-matrix between biological and SHM systems (cells with dots indicate matching features).

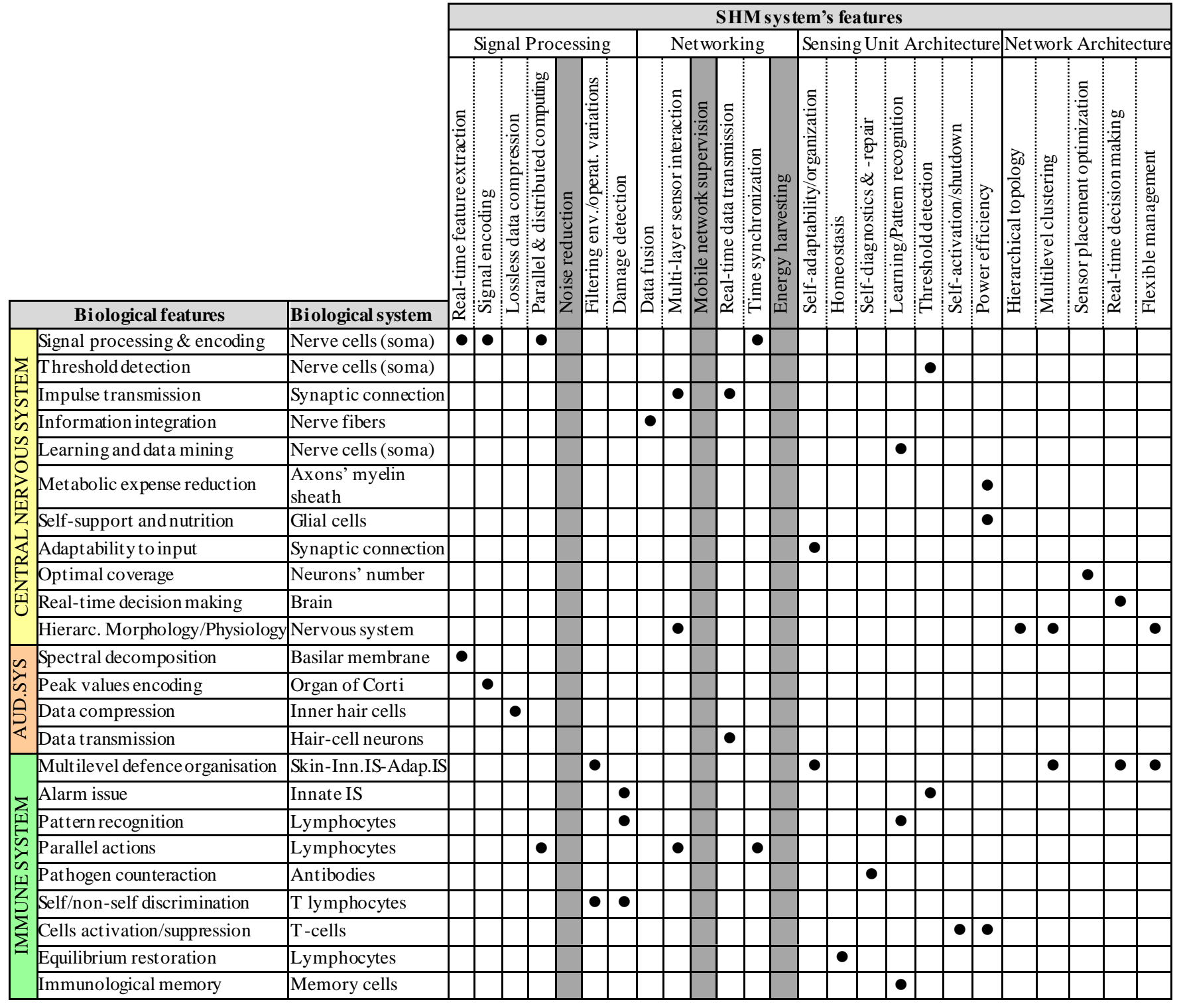

Taking this into account and based on the critical appraisal emerged from the previous paragraphs, a holistic framework tailored to SHM requirements is defined. This framework embraces both architectural and networking issues and is materialized into a compatibility-matrix where the desired SHM system's characteristics are distributed along the top and the biological features identified from the analysis of the three sources of bio-inspiration discussed in the paper - are arranged along the vertical axis 
(Table 1). Scope of the matrix is to help match analogous features between biological and SHM systems and to easily discriminate desired from unwanted nature-inspired characteristics. This pairing process will ultimately assist in the selection of the most compatible functions to transfer from nature to structural monitoring system. The stronger the compatibility (analogy), the stronger the future solution (synthesis).

Although limited, the proposed matrix can be considered as the first attempt to systematize a framework for bio-inspired SHM. Hitherto, only a few bio-inspired analogies have found actuation in SHM. Most gaps still have to be filled, thereby calling for further research. The next step will entail increasing the order of the matrix by finding a wider range of functional analogies. The ones showing the closest fit will be thoroughly analysed for possible implementation into biomimetic engineered systems for optimal vibration-based SHM.

\section{CONCLUSIONS}

A critical analysis of the current state-of-the-art in the field of structural health monitoring has been provided in this paper, including an overview of the recent developments in the area of biomimetic engineering for SHM purposes. The work has revealed that biomimetics still lacks a systematic approach. Apart from a few exceptions, most of bio-inspired paradigms and algorithms developed so far were conceived for reasons other than structuralmonitoring and they ultimately diverged from the primary source of inspiration. In order to go back to the original biological principles and overcome the conflicts of actual technologies, the authors have pointed out the need to tailor biomimetics to SHM requirements according to a holis tic approach. To achieve that, a compatibility-matrix is outlined to assist in the identification of functional analogies between SHM systems and biological kingdom, and to guide in the selection of the best solutions for future implementation into real engineered systems for robust structural monitoring and vibration analysis.

\section{ACKNOWLEDGMENTS}

This work was financed by FEDER funds through the Competitiveness Factors Operational Programme - COMPETE and by national funds through FCT - Foundation for Science and Technology within the scope of the project POCI-01-0145-FEDER007633.

\section{REFERENCES}

[1] Brownjohn, J.M.W. (2007). "Structural health monitoring of civil infrastructure." Philosophical Transactions ofthe Royal Society of London A: Mathematical, Physical and Engineering Sciences, 365(1851), 589-622.

[2] Spencer, B.F., Ruiz-Sandoval, M., and Kurata, N. (2004). "Smart sensing technology for structural health monitoring." In $13^{\text {th }}$ World Conference on Earthquake Engineering, Vancouver, Canada.

[3] Lynch, J.P. (2007). “An overview of wireless structural health monitoring for civil structures.” Philosophical Transactions of the Royal Society, Series A: Mathematical, Physical and Engineering Sciences, 365(1851), 345-372.

[4] Lynch, J.P., and Loh, K.J. (2006). "A summary review of wireless sensors and sensor networks for structural health monitoring." The Shock and Vibration Digest, 38(2), 91-130.

[5] Lei, Y., Kiremidjian, A.S., Nair, K.K., Lynch, J.P., and Law, K.H. (2005). "Algorithms for time synchronization of wireless structural monitoring sens ors." Earthquake Engineering and Structural Dynamics, 34(6), 555-573.

[6] Harkness, J.M. (2002). "A lifetime of connections - Otto Herbert Schmitt, 1913-1998." Physics in Perspective, 4(4), 456490.

[7] Velcro, S.A. (1955). Improvements in or relating to a method and a device for producing a velvet type fabric. Patent no. 721 338, Switzerland.

[8] Bechert, D.W., Bruse, M., Hage, W., and Meyer, R. (2000). "Fluid mechanics of biological surfaces and their technological application." Naturwissenschaften, 87(4), 157-171.

[9] Ayers, J., and Witting, J. (2007). "Biomimetic approaches to the control of underwater walking machines." Philosophical Transactions of the Royal Society of London, Series A: Mathematical, Physical and Engineering Sciences, 365(1850), 273-295.

[10] Kennedy, J. (2010). "Particle swarm optimization.” Encyclopedia of machine learning. Sammut C and Webb GI eds, Springer, 760-766, New York, NY, USA.

[11] Bonser, R.H. (2006). "Patented biologically-inspired technological innovations: a twenty-year view." Journal of Bionic Engineering, 3(1), 39-41.

[12] Bialek, W., Rieke, F., de Ruyter Van Steveninck, R.R., and Warland, D. (1991). "Reading a neural code." Science, 252(5014), 1854-1857.

[13] Kandel, C.E.R., Schwartz, J.H., and Jessell, T.M. (2000). Principles ofneural science, 4th ed., McGraw-Hill, New York.

[14] McCulloch, W.S., and Pitts, W.H. (1943). “A logical calculus of the ideas immanent in nervous activity." Bulletin of Mathematical Biophysics, 5(4), 115-133. 
[15] Hagan, M.T., Demuth, H.B., Beale, M.H., and De Jesús, O. (2014). Neuralnetwork design. 2nd ed., Martin Hagan.

[16] Anderson, J., and Rosenfeld, E. (1990). Neurocomputing: directionsfor research. MIT Press, Cambridge.

[17] Doebling, S.W., Farrar, C.R., Prime, M.B., and Shevitz, D.W. (1996). Damage identification and health monitoring of structural and mechanical systems from changes in their vibration characteristics: a literature review. Report LA-13070MS, National Laboratory, Los Alamos.

[18] Hecht-Nielsen, R. (1988). “Applications of counter propagation networks.” Neural Networks, 1(2), $131-139$.

[19] Jiang, S., Fu, C., and Zhang, C. (2011). "A hybrid data-fusion system using modal data and probabilistic neural network for damage detection." Advances in Engineering Software, 42(6), 368-374.

[20] Butcher, J., Day, C., Austin, J., Haycock, P., Verstraeten, D., and Schrauwen, B. (2014). "Defect detection in reinforced concrete using random neural architectures." Computer-Aided Civil and Infrastructure Engineering, 29(3), $191-207$.

[21] Fan, W., and Qiao, P. (2011). "Vibration-based damage identification methods: a review and comparative study." Structural Health Monitoring, 10(1), 83-111.

[22] Manning, R. (1994). "Damage detection in adaptive structures using neural networks." In 35th AIAA/ASME/ASCE/AHS/ASC Structures, Structural Dynamics, and Materials Conference, Hilton Head, SC, USA. Washington: American Institute of Aeronautics and Astronautics.

[23] Peckens, C., and Lynch, J.P. (2013). "Utilizing the cochlea as a bio-inspired compressive sensing technique." Smart Materials and Structures, 22(10), 105027.

[24] Yost, W.A. (1994). Fundamentals of hearing: an introduction. New York.

[25] Peckens, C., and Lynch, J.P. (2015). "Resource-efficient wireless sensor network architecture based on bio-mimicry of the mammalian auditory system.” Journal of Intelligent Material Systems and Structures, 26(1), 79-100.

[26] Peckens, C.A. (2014). Bio-inspired compressive sensing based on auditory neural circuits for real-time monitoring and control of civil structures using resource constrained sensornetworks. PhD Thes is, University of Michigan, USA.

[27] Janeway, Jr C.A., Travers, P., Walport, M., and Shlomchik, M.J. (2001). Immunobiology: the immune system in health and disease. 5th ed., Garland Publishing, New York.

[28] De Castro, L.N., Nunes, L., and Von Zuben, F.J. (1999). Artificial immune systems: Part I-basic theory and applications. Report TR - DCA 01/99, Universidade Estadual de Campinas, Brasil.

[29] Lederberg, J. (1959). “Genes and antibodies.” Science, 129(3364), 1649-1653.

[30] Burnet, F.M. (1976). "A modification of Jerne's theory of antibody production using the concept of clonal selection." CA: A Cancer Journal for Clinicians, 26(2), 119-121.

[31] De Castro, L.N., and Von Zuben, F.J. (2002). "Learning and optimization using the clonal selection principle." IEEE transactions on evolutionary computation, 6(3), 239-251.

[32] Dasgupta, D., Yu, S., and Nino, F. (2011). "Recent Advances in Artificial Immune Systems: Models and Applications." Applied Soft Computing, 11(2), 1574-1587.

[33] Jerne, N.K. (1974). "Towards a network theory of the immune system.” Annales d'immunologie, 125(1-2), $373-389$.

[34] Farmer, D.J., Packard, N.H., and Perelson, A.S. (1986). "The immune system, adaptation and machine learning." Physica D: Nonlinear Phenomena, 22(1), 187-204.

[35] Ishida, Y. (1990). "Fully distributed diagnosis by PDP learning algorithm: towards immune network PDP model." In 1990 IJCNN IEEE International Joint Conference on Neural Networks, San Diego, CA, USA.

[36] Aickelin, U., Dasgupta, D., and Gu, F. (2014). “Artificial immune systems.” In Search Methodologies: Introductory Tutorials in Optimization and Decision Support Techniques. Burke, E.K. and Kendall, G. eds, Springer, NY, USA.

[37] Bayar, N., Darmoul, S., Hajri-Gabouj, S., and Pierreval, H. (2015). "Fault detection, diagnosis and recovery using artificial immune systems:a review." Engineering Applications of Artificial Intelligence, 46, 43-57.

[38] Chen, B., and Zang, C. (2009). “Artificial immune pattern recognition for structure damage classification.” Computers and Structures, 87(21), 1394-1407.

[39] Abbasidoust, F., Lotfollahi-Yaghin, M., Sadeghi, M.H., Mojtahedi, A., Ettefagh, M.M., and Hassanzadeh, Y. (2012). “A robust damage detection method developed for offshore jacket platforms using modified artificial immune sys tem algorithm." China Ocean Engineering, 26(3), 379-395.

[40] Anaya, M., Tibaduiza, D.A., and Pozo, F. (2015). "A bioinspired methodology based on an artificial immune systemfor damage detection in Structural Health Monitoring." Shock and Vibration, 2015.

[41] Yi, T.H., Li, H.N., and Zhang, X.D. (2015). "Health monitoring sensor placement optimization for Canton Tower using immune monkey algorithm." Structural Control and Health Monitoring, 22 (1), 123-138.

[42] De Castro, P.A.D., and Zuben, F.J. (2009). "A Bayesian artificial immune systemfor the effective handling of building blocks.” Information Sciences, 179(10), 1426-1440. 